\title{
Infrared Emission Spectrum of Silicon Carbide Heating Elements
}

\author{
James E. Stewart ${ }^{1}$ and Joseph C. Richmond
}

\begin{abstract}
A method for determining the spectral emittance of a silicon carbide heating element (Globar) was devised and data were obtained over the wavelength range 1.25 to $15.25 \mu$ for four different temperatures. The spectral emittance at all four temperatures, $482^{\circ}, 649^{\circ}$, $816^{\circ}$, and $982^{\circ} \mathrm{C}\left(900^{\circ}, 1,200^{\circ}, 1,500^{\circ}\right.$, and $\left.1,800^{\circ} \mathrm{F}\right)$, was found to be approximately constant except for a gradual rise in emittance from 1.25 to about $4 \mu$ and two minimums in the curves at longer wavelengths. The first of these minimums, which occurred at about $9 \mu$, was ascribed to minor amounts of $\mathrm{SiO}_{2}$ present on the surface of the silicon carbide heating element, whereas the second at about $12.5 \mu$ corresponds to one of the strong Raman lines for $\mathrm{SiC}$.
\end{abstract}

\section{Introduction}

There is much current interest in heat-transfer processes at high temperatures. The extreme temperatures encountered in jet aircraft engines have long presented problems; but in modern aircraft performing at supersonic velocities even skin temperatures are well into the range where metals show undesirable creep and corrosion properties. Because radiative processes play a primary role in the transfer of heat to and from such parts it is desirable to obtain data on the thermal emission of various metals and coatings of engineering interest in a temperature range from $482^{\circ}$ to $982^{\circ} \mathrm{C}\left(900^{\circ}\right.$ to $\left.1,800^{\circ} \mathrm{F}\right)$.

Some work has been done in measuring the total emittances of various materials of interest. Total emittance, the ratio of the total radiant energy per unit area emitted by a specimen to that emitted by a black body at the same temperature, is not a satisfactory measure of the amount of radiant energy emitted or absorbed by a specimen for use in heattransfer computations because (1) the spectral distribution of the radiant energy emitted by all surfaces, including black bodies, varies with temperature, (2) the spectral distribution of the radiant energy emitted by a surface may be markedly different from that of a black body at the same temperature, and (3) air and other gases absorb radiant energy at certain wavelengths that are characteristic of the gas.

Spectral emittance is the ratio of the radiant energy per unit area emitted by a surface at a given wavelength to that emitted by a black body at the same temperature and wavelength, for all wavelengths at which the specimen or black body emits a significant amount of energy. The radiant energy transmitted from a hot to a cold surface can be computed if the temperature of both surfaces, their spectral emittances at their respective temperatures, and the spectral absorption of any material through which the radiant energy passes between the surfaces, are known.

* This project was under the sponsorship and with the financial assistance of the National Advisory Committee for Aeronautics.

Present address: Beckman Instruments, Inc., Fullerton, Calif.
Absolute measurements of infrared radiation are difficult to make. The spectral distribution of energy emitted by a black body as a function of temperature has been known for many years, hence most emission measurements today are referred to a black body as standard. By definition, black bodies absorb all radiation incident upon them, and emit according to the Planck radiation law. A cavity with its walls at uniform temperature, having an appropriately small opening, furnishes a close approximation to the required conditions, and is not only the best standard for laboratory use, but also, under the proper conditions, is very nearly ideal in its physical characteristies. However, a small opening focused on a spectrometer requires that the spectrometer slit also be kept small, and this in turn requires a high amplifier gain and slow scanning speeds. It also makes focusing more difficult. To circumvent these difficulties it was decided to measure the spectral emission of the various test surface with respect to a secondary standard and then to adjust the resulting data to emittance after measuring the secondary standard against a black body.

A recrystallized silicon carbide rod (Globar) was believed suitable as the secondary standard because of its ruggedness, resistance to oxidation, and the ease with which the temperature can be controlled when the material is resistance heated by passing a current through it.

The present paper describes the method used for measuring the spectral emittance of test bodies and for calibrating the Globar, used as a secondary standard, to a sufficient degree of accuracy to permit the comparison of emission properties of test bodies. Values given below for the spectral emittance of Globar are not to be taken as standard emittance data for silicon carbide for reasons to be discussed.

\section{Review of Earlier Work}

The total emittance of silicon carbide powder bonded with 25 percent of clay was reported by Pirani $[1]^{2}$ to be nearly 1.0 at $200^{\circ} \mathrm{C}$, dropping to about 0.75 at $900^{\circ} \mathrm{C}$. The spectral emittance of a

\footnotetext{
${ }^{2}$ Figures in brackets indicate the literature references at the end of this paper.
} 
silicon carbide heating element (Globar) was first reported by Silverman [2] for the range 2 to $15 \mu$ and for a single temperature of $1,102^{\circ} \mathrm{C}$ (approximately $\left.2,015^{\circ} \mathrm{F}\right)$. Silverman used a specially prepared hollow cylinder of silicon carbide with a large outside diameter. An axial slot cut through the wall permitted radiation from the cavity to be compared with radiation from the surface of the source. His emittance curve shows a gradual rise from about 0.73 at $2 \mu$ to about 0.84 at $5 \mu$, a fairly constant level to about $9.4 \mu$ where a shallow minimum occurs followed by a stronger minimum at about $12.5 \mu$. He identified the minimum and maximum of the emittance curve at about 12.5 and $10 \mu$ with the maximum and minimum, respectively, of the reflectivity curve at 12 and $10 \mu$.

Brügel [3] measured the spectral emittance of a standard spectrometer source of silicon carbide (Globar) in the 0.6 to $15 \mu$ region for a series of temperatures from $727^{\circ}$ to $1,527^{\circ} \mathrm{C}$ (approximately $1,340^{\circ}$ to $2,780^{\circ} \mathrm{F}$ ), with a comparison cavity black body at $727^{\circ} \mathrm{C}$ and instrument deflections calculated from those obtained at $727^{\circ} \mathrm{C}$ for measurements at other temperatures. He shows an emittance curve for $970^{\circ} \mathrm{C}\left(1,778^{\circ} \mathrm{F}\right)$ which rises from 0.75 at $2 \mu$ to a maximum of about 0.87 at $8 \mu$ and falls to about 0.77 at $15 \mu$. His curve shows a minimum at about $12.2 \mu$ but none near $9.4 \mu$, probably because his measurements were made at $0.5 \mu$ intervals. Brügel reports a linear temperature dependence of spectral emittance ranging from about $3 \times 10^{-5}$ per degree Kelvin at $2 \mu$ and $14 \mu$ to a maximum of about $12 \times 10^{-5}$ per degree Kelvin at $10 \mu$.

The reflectance spectrum of silicon carbide was first measured by Coblentz [4] and repeated by Schaefer and Thomas [5], who also measured the infrared absorption spectrum of a plate. The reflectance spectrum showed only the strong peak at $12 \mu$ and the minimum at $10 \mu$ mentioned above.

Ramdas [6] recently redetermined the absorption spectrum between 1 and $20 \mu$ for a number of modifications of silicon carbide, using plates ranging between 0.14 and $1.0 \mathrm{~mm}$ in thickness. He found a very strong band probably centered near $12 \mu$ and numerous weaker combination tones. Ramdas assigns the $12 \mu$ band to a vibration mode involving an asymmetric stretching of $\mathrm{C}-\mathrm{Si}$ bonds.

The Raman spectrum of silicon carbide has been observed by Narayanan [7], who reported a strong line at $818 \mathrm{~cm}^{-1}(12.2 \mu)$.

\section{Experimental Method}

A Perkin-Elmer model 21 double-beam infrared spectrophotometer [8] was used with a sodium chloride prism for all measurements. Ordinarily the Perkin-Elmer instrument is used for transmission measurements and is arranged as shown schematically in figure 1. A Nernst glower source, $\mathrm{N}$, is viewed by two pairs of mirrors which produce images, magnified two times on the monochromator slits. The instrument records the ratio of the radiance ${ }^{3}$ of the two images and this ratio depends

\footnotetext{
${ }^{3}$ Radiant flux per unit surface area.
}

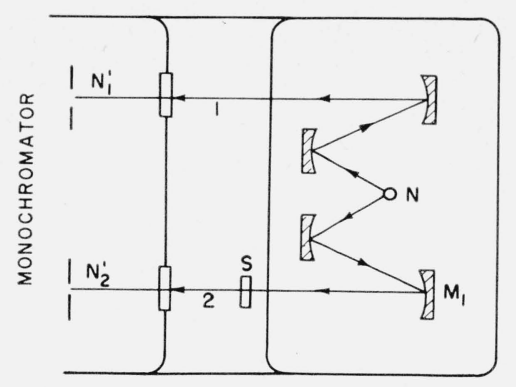

Figure 1. Schematic drawing of double-beam infrared spectrophotometer when arranged for transmission measurements.

$\mathrm{N}$, Nernst glower source; $\mathrm{M}$, mirror; $\mathrm{S}$, specimen.

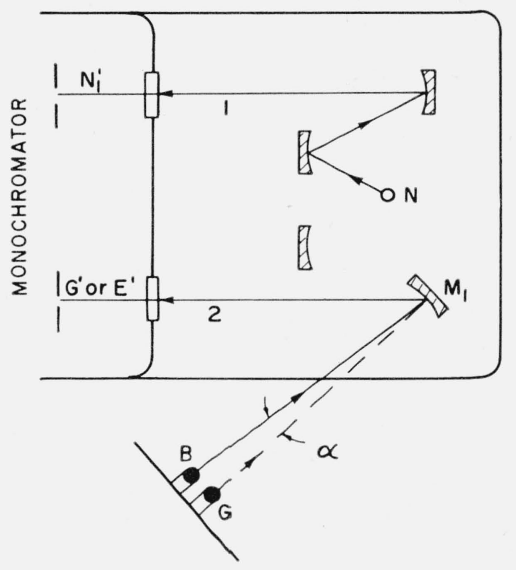

Figure 2. Same instrument as in figure 1 as modified for measuring the relative emission from a black body $(B)$ as compared to a Globar $(G)$.

on the absorption of a transmission specimen placed at $\mathrm{S}$.

For emission measurements the equipment is modified as shown schematically in figure 2 . The mirror, $\mathbf{M}_{1}$, is rotated as indicated so as to produce an unmagnified image of either the Globar source, $\mathrm{G}$, or the black body, B, on the slit. ${ }^{4}$ Because the path lengths of the two beams are approximately the same, the absorption by atmospheric water vapor and carbon dioxide will be nearly balanced.

The design of the black body is shown in figure 3 . The core is made of graphite and the two electrodes (not shown in the figure), one at each end of the core, are connected to a welding transformer of variable power input. During a test, nitrogen is permitted to flow into the Vycor cylinder surrounding the cavity in the graphite. This prevents rapid oxidation of the graphite. An asbestos baffle serves to prevent the nitrogen from impinging directly on the graphite, which might otherwise produce a cool spot at this area.

${ }^{4}$ The images of both the Globar and the opening in the black body are sufficiently large to fill the slit and hence require no magnification. 


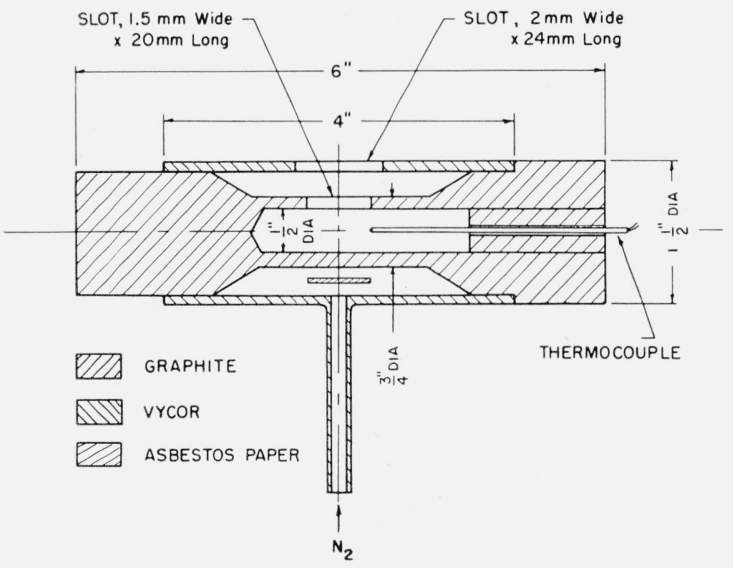

FIG URE 3. Design of black-body source used for measuring spectral emittance of silicon carbide heating element.

Nitrogen flowing through Vycor cylinder prevents rapid oxidation of graphite during determinations at elevated temperatures.

The temperature at the inside of the cavity of the graphite core was measured with a Chromel-Alumel thermocouple. During operation neither the thermocouple bead nor the ceramic insulator could be observed through the slit, thus indicating good temperature uniformity within the cavity. In each test the temperature was maintained within $\pm 1.7^{\circ} \mathrm{C}$ of that desired by manually adjusting the power input.

The temperature of the silicon carbide heating element was measured by a Chromel-Alumel couple inserted into a hole that was drilled into the side of the element with a Cavitron [9]. The temperature of this element was also maintained to within $\pm 1.7^{\circ} \mathrm{C}$ of that desired.

In making a determination, the silicon carbide element and graphite core were brought to the same temperature. Mirror $\mathrm{M}_{1}$ in figure 2 was adjusted so as to focus the image of the element on the slit of the monochromator and a trace representing emission with respect to the Nernst glower source was obtained over the wavelength region 1.25 to $15.25 \mu$. Next, mirror $\mathrm{M}_{1}$ was rotated slightly so as to fill the slit with radiation from the black body and a second trace was obtained. The ratio of the heights of the two curves at any point, corrected for the difference introduced by the slight shift in angle of mirror $\mathrm{M}_{1}$, was then taken as the emittance of the silicon carbide surface at that particular wavelength. In making these calculations, the angular variation in reflectivity of the aluminum coating on the mirror as well as the effect of changing optical aberrations with angle were assumed negligible for the small changes in angles involved.

\section{Results and Discussion}

Figure 4 is a plot of the emittance of silicon carbide as a function of the wavelength of emitted energy for $482^{\circ}, 649^{\circ}, 816^{\circ}$, and $982^{\circ} \mathrm{C}\left(900^{\circ}, 1,200^{\circ}, 1,500^{\circ}\right.$, and $\left.1,800^{\circ} \mathrm{F}\right)$. The results are in qualitative agreement with those of Silverman [2] and of Brügel [3],

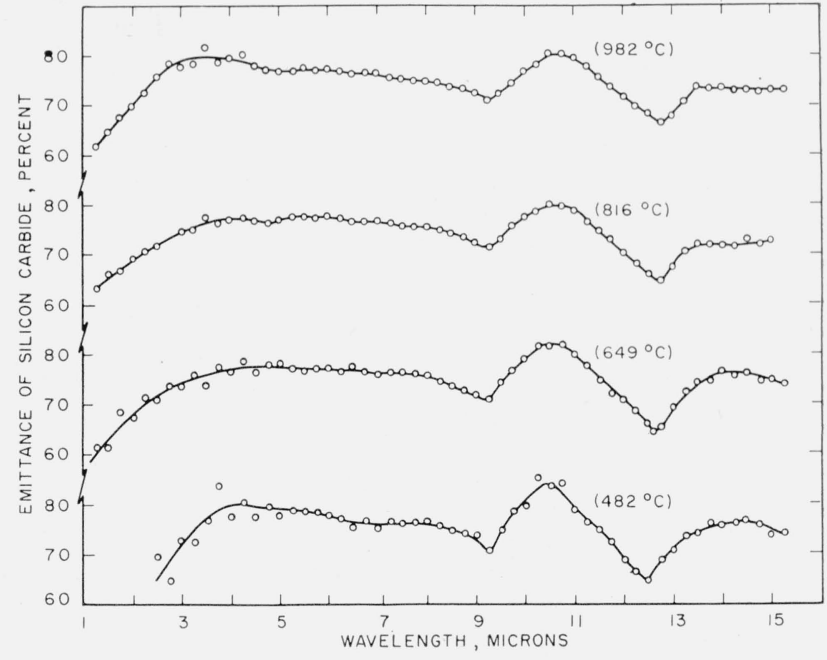

Figure 4. Spectral emittance of Globar heating element at four temperatures.

but the values tend to be somewhat lower than those reported by these two investigators. The change in spectral emittance with temperature is small; therefore, the lower values obtained in the present series of measurements are probably not due to differences in the range of temperatures in the three laboratories.

The measurements were reproducible within a range of about \pm 2 percent at the shorter wavelengths $(\lambda<5 \mu)$ and about \pm 1 percent at the longer wavelengths. Fluctuations in the temperature of the Nernst glower are probably responsible for much of the uncertainty. Within these experimental limits no temperature dependence of emittance was found for regions sufficiently remote from the minimums near 9 and $12 \mu$. The minimums however, were definitely temperature sensitive. As seen in figure 4, they shift to longer wavelengths as the temperature is increased, and in fact appear to do so as linear functions of temperature: $\lambda_{1}=8.97$ $\left(1+4.1 \times 10^{-5} \mathrm{~T}\right)$ and $\lambda_{2}=12.17\left(1+5.08 \times 10^{-5} \mathrm{~T}\right)$. ( $T$ =temperature in ${ }^{\circ} \mathrm{K}$.) This temperature dependence was investigated further by determining the positions of the peaks at additional temperature of $193^{\circ}, 260^{\circ}, 316^{\circ}, 398^{\circ}$, and $1,115^{\circ} \mathrm{C}\left(380^{\circ}, 500^{\circ}\right.$, $600^{\circ}, 749^{\circ}$, and $\left.2,039^{\circ} \mathrm{F}\right)$. The broadness of the peaks, particularly at the lower temperatures, makes accurate determinations of the minimums difficult. It is clear, however, that the $12 \mu$ peak should occur at about $12.2 \mu$ at room temperature, in excellent agreement with the Raman observation. The other peak should be found at about $9.0 \mu$ at room temperature. McMahon [8] has observed a similar temperature shift of the peak observed near $9.5 \mu$ in the emission spectra of glasses. He attributes this to an increase in the $\mathrm{Si}-\mathrm{O}$ bond distance as a result of thermal expansion.

Because no band had been observed at $9 \mu$ in the infrared reflection or absorption spectra, or in the Raman spectra of silicon carbide, the appearance of a peak there in the emission spectra is puzzling 
Both Ramdas [6] and Schaefer and Thomas [5] made their observations on plates oriented with the basal plane perpendicular to the incident radiation. Hence vibrations perpendicular to the basal plane might not be observed [10]. An attempt was made to observe the infrared absorption spectrum of a randomly oriented sample of silicon carbide by suspending some very fine powder in a potassium iodide disk. The differences in refractive indices (about 1.65 for KI and 2.6 for silicon carbide at $750 \mathrm{m \mu}$ ) were too great to permit observation of a good spectrum. A broad band was observed at about $12 \mu$ and a Christiansen window [11] occurred at $9.5 \mu$, no doubt where the refractive indices of silicon carbide and potassium iodide coincide. No band was found at $9 \mu$.

A plausible explanation of the $9 \mu$ band is that it arises from an impurity. The various forms of silica are known to have strong reflection bands near $9 \mu$, due to an asymmetric stretching of the $\mathrm{Si}-\mathrm{O}-\mathrm{Si}$ bonds. Furthermore, these bands seem to shift to longer wavelengths as temperature is increased [12]. Silica is a likely impurity in silicon carbide because the material is prepared by reduction of silica with carbon at high temperature and also because X-ray $[13,14]$ and electron diffraction [13] studies have shown that cristobalite is formed by oxidation of silicon carbide at temperatures encountered by heating elements in ordinary use. $\beta$-Cristobalite, the high temperature form, has its reflection maximum at about $8.8 \mu$ at $300^{\circ} \mathrm{C}$ [12]. Because a peak in the reflection curve is influenced by both the refractive index and the absorption coefficient, it does not necessarily coincide with a vibrational frequency of the material. The true frequency of the vibration producing the $8.8 \mu$ reflection peak in the $\beta$-cristobalite has not been measured, but by analogy with $\alpha$-cristobalite and quartz it probably lies at a wavelength longer than $8.8 \mu$ by a few tenths of a micron. In addition, the bonding material in Globar is likely to produce its own emission peaks.

No change in the cristobalite peak, or in the entire Globar spectrum, was detected during the period of time (about $50 \mathrm{hr}$ ) these and other measurements required. Nevertheless, because of the known changes in emitting properties of Globar sources with time it is necessary to compare the Globar with the black body at intervals during a series of measurements.

The discrepancy between the results reported here and the earlier observations could be due to differences in the composition or aging of the specimens studied or to experimental difficulties associated with temperature equilibrium and measurement. The spectral emittance of the Globar used in this study was checked in the region between 1.0 and $2.5 \mu$ by R. G. Johnston and E. D. Tidwell, using a spectrometer and black body furnace of the Radiometry Section of the Bureau. Agreement was obtained throughout the region only by adjusting the temperature of the Globar to obtain agreement at $2.5 \mu$. The instrument used had very short slits $(3 \mathrm{~mm})$ which permitted the observation of energy emitted from different parts of the Globar. In this way the existence of a steep thermal gradient along the Globar was demonstrated, with the ends of the Globar perhaps as much as 10 percent cooler than the middle. An averaged intensity is observed with a spectrometer having longer slits.

The peak in Brügel's curve showing the temperature rate of change of emittance as a function of wavelength is produced, or at least augmented, by the shift in the 9 and $12 \mu$ peaks. The observations by Pirani [1] that total emittance of a mixture of silicon carbide and clay decreases with increasing temperature is consistent with a spectral emittance that is independent of or even slightly increasing with temperature, for as the temperature is increased the peak of the black body emission curve moves to shorter wavelengths where the emittance of silicon carbide is decreasing. The clay in Pirani's sample also undoubtedly affects the emission properties.

As the purpose in measuring the spectral emittance of the silicon carbide heating element is to be able to determine the emittances of coated metal specimens, a typical example of this application is shown in figure 5. The upper curve is the emission, normal to the surface, from a sand-blasted stainless-steel strip coated with A-418, a material consisting of chromic oxide, barium borosilicate glass, and clay, relative to silicon carbide at $982^{\circ} \mathrm{C}\left(1,800^{\circ} \mathrm{F}\right)$. The lower curve is the emittance adjusted to be relative to a black body.

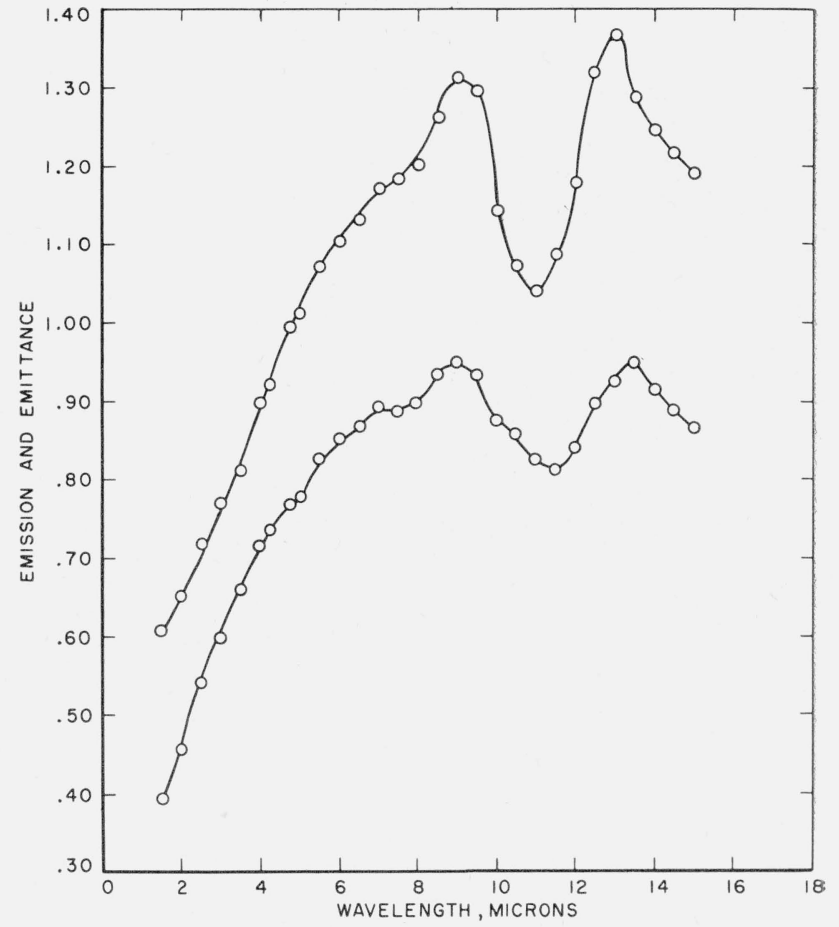

Figure 5. Spectral emission (upper curve) and spectral emittance (lower curve) of a barium borosilicate coating on stainless steel.

Coating A-418 on stainless steel. $982^{\circ} \mathrm{C}\left(1,800^{\circ} \mathrm{F}\right)$. 


\section{Conclusion}

The spectral emittance of silicon carbide seems to be independent of temperature in the range $482^{\circ}$ to $982^{\circ} \mathrm{C}\left(900^{\circ}\right.$ to $\left.1,800^{\circ} \mathrm{F}\right)$ within the experimental error of the measurements, except for two minimums near 9 and $12.2 \mu$. The wavelengths of these peaks appear to vary nearly linearly with temperature and to fall at longer wavelengths at higher temperatures. The peak near $12.2 \mu$ undoubtedly arises from a vibration of the silicon carbide itself, whereas the peak near $9 \mu$ probably is contributed by cristobalite which is known to form when silicon carbide oxidizes.

The observed variation in emission from different regions along the Globar rod leads to the conclusion that a Globar source is not a precise standard for emissivity measurements unless the emittance from a fixed portion of its surface is determined by comparison with a black-body source.

The authors express their gratitude to R. G. Johnston, E. D. Tidwell, and E. K. Plyler of the Radiometry Section, and to D. G. Moore and W. N. Harrison of the Enameled Metals Section for helpful discussions and assistance.

\section{Appendix. Emission, Emittance, and Emissivity}

The use of the words emission, emittance, and emissivity in this paper follows the recommendations of Worthing [15].

Emission is the act or process by which radiant energy is emitted by a body as a consequence of its temperature only. This term is also used for the rate of such emission in units of energy per unit area and unit time. It is influenced by the composition, thickness, and surface texture of the specimen.

Emittance is a property of a specimen, regardless of its composition, thickness, or surface texture, and is the ratio of the emission of the specimen to that of a black body at the same temperature.

Emissivity is a property of a material, and is the emittance of a specimen of the material having a polished surface, of sufficient thickness to be opaque. It represents a minimum value of emittance as regards the effect of surface texture, and a maximum value as regards the effect of thickness (opacity) of the specimen.

\section{References}

[1] M. Pirani, Radiation properties of different substances within the temperature range $250^{\circ} \mathrm{C}$ to $800^{\circ} \mathrm{C}$, J. Sci. Inst. 16, 372 (1934).

[2] S. Silverman, The emissivity of Globar, J. Opt. Soc. Am. 38, 989 (1948).

[3] W. Brügel, Strahlungsmessungen an Elektrisch Geheitzen SiC-Stäben, Z. Physik 12\%, 400 (1950).

[4] W. W. Coblentz, Radiometric investigations of infrared absorption and reflection spectra, Bul. BS 2,457 (1906) S45.

[5] C. Schaefer and M. Thomas, Oberschwingungen in Ultraroten Absorption-spektren, Z. Physik 12, 330 (1923).

[6] A. K. Ramdas, The infrared absorption spectrum of silicon carbide, Proc. Indian Acad. Sci. [A] 37, 571 (1953).

[7] P. S. Narayanan, Raman spectrum of Carborundum, Current Sci. 21, 239 (1952).

[8] H. O. McMahon, Thermal radiation characteristics of some glasses, J. Am. Ceram. Soc. 34, 91 (1951).

[9] G. H. DeGroat, Soft steel and ultrasonics machine carbide, Am. Machinist 96 (19) 141 (September 15, 1952).

[10] J. E. Stewart, Use of partially oriented solid samples and unpolarized radiant energy in the analysis of infrared absorption spectra, J. Chem. Phys. 23, 986 (1955).

[11] R. B. Barnes and L. G. Bonner, The Christiansen filter effect in the infrared, Phys. Rev. 49, 732 (1936).

[12] I. Simon and H. O. McMahon, Study of the structure of quartz, cristobalite, and vitreous silica by reflection in infrared, J. Chem. Phys. 21, 23 (1953).

[13] G. Heine and P. Scherer, Untersuchung der Oberflächenschicht von Siliciumcarbid und Umwandlung von SiC in Cristobalit, Helv. Phys. Acta 13, 489 (1940).

[14] T. H. Elmer, Silicon carbide heating elements-development, uses, chemical and physical investigations, Bul. Am. Ceram. Soc. 32, 23 (1953).

[15] A. G. Worthing, Temperature radiation emissivities and emittances. Temperature, Its Measurement and Control in Science and Industry, p. 1164 (American Institute of Physics, 1941).

Washington, December 3, 1956. 